\title{
Attention Analysis in Interactive Software for Children with Autism
}

\author{
A. Ould Mohamed \\ aouldm01@univ-Ir.fr \\ L3I, Université de La Rochelle \\ Av Michel Crépeau, 17042 \\ La Rochelle, France
}

\author{
V. Courboulay \\ vcourbou@univ-Ir.fr \\ L3I, Université de La Rochelle \\ Av Michel Crépeau, 17042 \\ La Rochelle, France
}

\author{
K. Sehaba, M.Menard \\ ksehaba, \\ m.menard@univ-Ir.fr \\ L3I, Université de La Rochelle \\ Av Michel Crépeau, 17042 \\ La Rochelle, France
}

\begin{abstract}
This work is a part of an ongoing project that focuses on potential applications of an interactive system that helps children with autism. Autism is classified as a neurodevelopmental disorder that manifests itself in markedly abnormal social interaction, communication ability, patterns of interests, and patterns of behavior [1]. Children with autism are socially impaired and usually do not attend to the people around them. An interesting point which characterized children with autism is that they are unable to choose which event is more or less important. As a consequence they are often saturated because of too many stimuli and thus they adopt an extremely repetitive, unusual, self-injurious, or aggressive behaviour. Recently, a new trend of using human computer interface (HCI) technology and computer science in the treatment of autism has emerged $[2,3]$. The platform we developed helps children with autism to focus their attention on a specific task. In this article, we only present the attention analysis system which is a part of a more general system that used a multi-agent architecture [4]. Each task proposed on our system fit to each child, is reproducible and evolutive following a specific scenario defined by the expert. This scenario takes into account age, ability, and degree of autism of each child. In order to focus a child's attention onto the relevant object, our system displays or plays specific stimulus; once again the specific stimulus is defined for each child. Symbol or sound represents an emotional and satisfaction value for the child. The major problem is to define the correct moment when the system has to (dis)play this signal. We tackle this problem by defining a robust measure of attention. This measure is defined by analyzing the gaze direction and the face orientation, and incorporating the child's specific profile. Following expert directives, our system helps children to categorize elementary perception (strong, smooth, quick, slow, big, small...). Our objective is that children re-use these classifications in others situations.
\end{abstract}

\section{Categories and Subject Descriptors}

I.2 [Vision and Scene Understanding]: Scene analysismotion; J.4 [Social and Behavioral science]: Psychology

\section{General Terms}

This section is limited to the following 16 terms: Algorithms, Management, Measurement, Documentation, Performance, Design, Economics, Reliability, Experimentation, Security, Human Factors, Standardization, Languages, Theory, Legal Aspects, Verification.

\section{Keywords}

Attention analysis, behavior study, children with autism

\section{INTRODUCTION}

Autism is not a disease, but a developmental disorder of the brain function. People with classical autism show three types of symptoms [1] :

- impaired social interaction,

- problems with verbal and non-verbal communication and imagination,

- and unusual or severely limited activities and interests.

Symptoms usually appear during the first three years of childhood and continue throughout life, since there is no cure. People with autism have a normal life expectancy. The hallmark feature of autism is impaired social interaction. Children with autism often have difficulty in interpreting tone of voice or facial expressions and do not respond to others' emotions. Many children with autism engage in repetitive movements or in self-injurious behaviour. People with autism often have abnormal responses to sounds, touch, or other sensory stimulation. They also may be extraordinarily sensitive to other sensations. These unusual sensitivities may contribute to behavioural symptoms such as resistance to being cuddled.

Appropriate management may favor relatively normal development and reduce undesirable behaviours, even if autism degree presents a great deal in severity. This behaviour may persist over time, thus it is very difficult to live with peo- 
ple with autism, to treat ${ }^{1}$ and teach them. The most severe cases are marked by extremely repetitive, unusual, selfinjurious, and aggressive behaviour. Other forms of autism include a personality disorder associated with a perceived learning disability. In spite of numerous signs, evaluation methods have been developed [5] :

- Psychological and behavioral treatment helps autistic children to approach the notion of the other, enabling them to build and re-use specific mental constructions to control their surrounding.

- the TEACCH method (Treatment and Education of Autistic and Related Communication-handicapped Children). TEACCH is not a single approach and even less a method [6]. It is a state program that tries to respond to the needs of autistic people using the best available approaches and methods known so far for educating them and to provide the maximum level of autonomy that they can achieve.

- Facilitated communication is an alternative means of expression for people who cannot speak, or whose speech is highly limited, and who cannot point reliably. The method has been used as a means to communicate for individuals with severe disabilities, including persons with labels of mental retardation or of course autism $[7]$.

In this last item, we find scientific works supporting the idea that computer science can be used in the field of autism treatment. For example, Gepner [8] deals with the difficulty for children with autism to perceive rapid motion and Fabri [9] deals with the use of emotionally expressive avatars in emotions encoding, etc. As a consequence, computer science becomes a research area in the structuration of people with autism. At this step, it is appropriate to present the next scheme representing cascade leading to mental disorders [8].

We focus our scientific effort on the bottom left part of the scheme. In the next section, we present the AutiSTIC project on which we work, but also the specific work of visual attention analysis.

\section{THE AUTISTIC PROJECT}

Several robotic platforms are developed as interactive playmates for children $[10,9,11,12]$. [13] uses virtual environments in teaching strategy, [14] is interested in the design of Human-computer Interfaces for children with autism. However, to our knowledge, there is no model that proposes an adaptive approach that takes into account the experts directives in an educational context. The modelling of this approach requires modelling of the knowledge of experts, the user profile and the dynamic of their interactions.

The project that we carry out, called AutiSTIC Project, is in partnership with the psychiatric service for children with

\footnotetext{
${ }^{1}$ We have to take care of the meaning of treatment, we means special education, adaptative care in order to give support to child and reduce consequence for family and people around him.
}

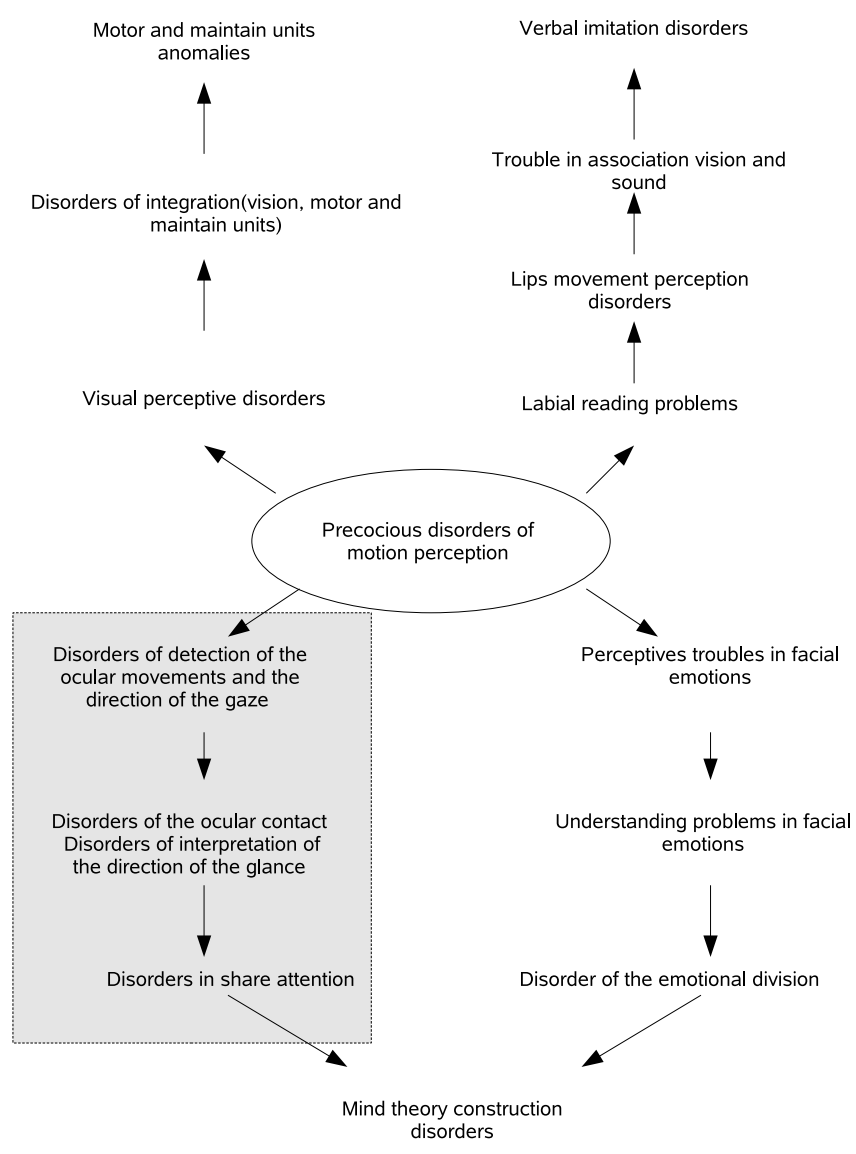

Figure 1: Synthetic cascade leading to mental disorders.

autism of La Rochelle hospital. Our objective is to implement a software and hardware system that can help autistic children during the rehabilitation process. It consists in establishing a multimode and multimedia dialogue between the assisted child and the system and helps him to be focused on his task during a computer game or exercice. The main goal is to help the child to improve his understanding about himself and his surrounding.

The role of such a system is to provide the child with the personalized activities in form of educational games. During a session, the system collects by various devices (camera, touch screen, mouse, keyboard...) the child's reactions, in order to understand his behaviour. The system responds in real time to the child's reactions using adequate actions that consider the expert's directives. These directives concern rupture, avoidance, stereotype gestures... For instance, the system may attract the child's attention by posting of a familiar image, or by playing a specific music.

Each child is characterized by specific competences and preferences, so he requires an adapted treatment. It is impossible to generalize activities without precaution, but we have to favor adaptability of system to take into account specific deficits observed for each child. It is important to locate 
and interpret carefully these intrinsic behaviours, in order to help him/her to rehabilitate. In the application context, our architecture aims to bring flexibility and modularity in the individualized rehabilitation of children with autism.

To summarize, in the AutiSTIC project, we develop specific tools which mainly allow to :

- provide software which can evaluate the degree of attention of each child and understand his own adaptative strategies ;

- be able to modify the child's belief by presenting virtual and interactive environment. This one must integrate autistic symptoms, for example the difficulty to perceive rapid motions ;

- emphasize the need of "same" which makes feel secure, but also interate different elements to avoid repetition and stereotypy.

We present here a part of our platform which observe children and analyse his visual attention.

\subsection{Observed behaviors}

Behaviors we are interested in during a game session are mainly characteristics of attention. Figure 2 presents the general diagram of implementation of game intended for child with autism. Control is done by observing and analyzing his behavior. To begin, the game presents stimuli at screen, then the system collects the child's reactions with respect to such stimuli. The behaviors which carry a particular interest initially relate to the rupture and the avoidance:

- rupture is defined as the removal of the child from the computer during a game session. Rupture, is a behavior which indicates the inadequacy between activity and child. In this specific case, our platform must start another activity.

- Avoidance is defined as the non reaction of the child to an activity. There exist two types of avoidance, temporal and visual.

- temporal avoidance means passivity during a certain time in front of the screen ;

- visual avoidance occurs when the child's gaze is not oriented to the screen ;

Avoidance occurs mainly due to an inadequacy between stimulus and child. In this case, our system must change either stimulus or game objective.

Thus, gaze and face orientations are precious signs in order to qualify and quantify attention. In the next section, we will specify in detail what is meant by attention. Indeed, there exist several types of attention as awakening and selective attention.

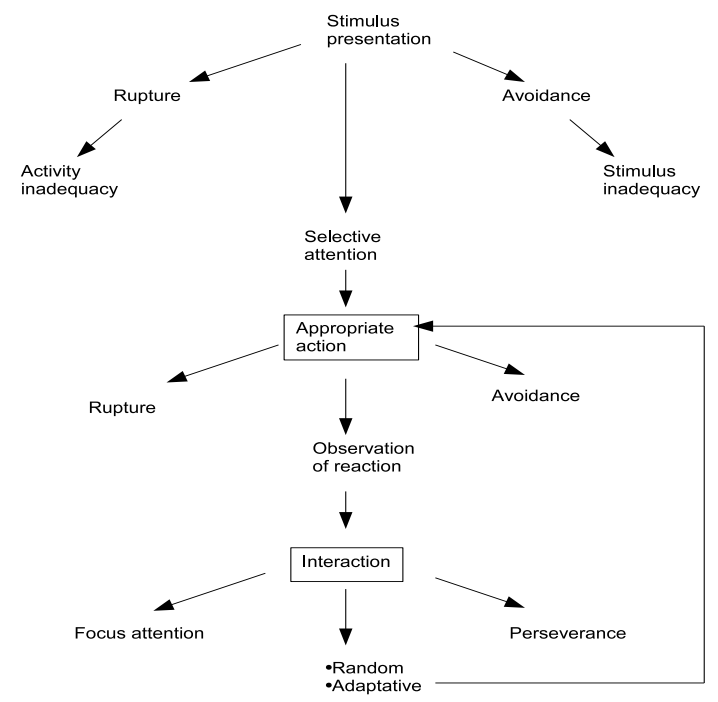

Figure 2: Behaviors analysis control 


\subsection{Attention}

In this section, we present different types of attention, following psychology and cognitive neuroscience classification [15]. Usually, attention is defined as the cognitive process of selectively concentration on one thing while ignoring other things.

- Awakening : becoming awake is a metabolic state which is characterized by consciousness, the opposite of sleep, in this state, we are sensory open to the world.

- Selective attention: A type of attention which involves focusing on a specific aspect of a scene while ignoring other aspects. Selective attention can be conscious (as when one chooses to attend to an interesting object instead of a less interesting one) or unconscious. An interesting point which characterized children with autism is that they are unable to choose which event is more or less important. As a consequence they are often saturated because of too many stimuli and thus they adopt an extremely repetitive, unusual, selfinjurious, or aggressive behaviour.

- Maintained attention : this kind of attention appears after the previous one. It is the activity keeper, allowing focusing and avoiding distraction.

- Shared attention : skill to treat two or more different types of information (sound, motion, action...)

- Internal or external absent-mindedness : internal or external preoccupation so great that the ordinary demands on attention are ignored.

- Vigilance is the act of watching for something to happen, of watching for danger. It is not something that humans are very good at, since it is difficult to maintain attention for very long without some stimulus.

For our AutiSTIC project, we are interested in selective and maintained attention. The analysis of the first one allows to know whether the child is involved in the activity. The second one enables us to assess the success of the game. In the next section, we present our architecture and more particularly the vision process developed to analyse and study previous attentions.

\section{SYSTEM ARCHITECTURE AND VISION PROCESS}

\subsection{System architecture}

As we already explained, each child is characterized by his own competences and preferences, so he requires an adapted treatment. Thus it is impossible to generalize activities without precaution. We have to favor adaptability of system to take into account specific deficits observed for each child. It is important to locate and interpret carefully specific behaviours as attention, in order to help him/her to rehabilitate. In the application context, our architecture aims to bring flexibility and modularity in the individualized rehabilitation of children with autism. Accordingly, we propose a system architecture which allows:

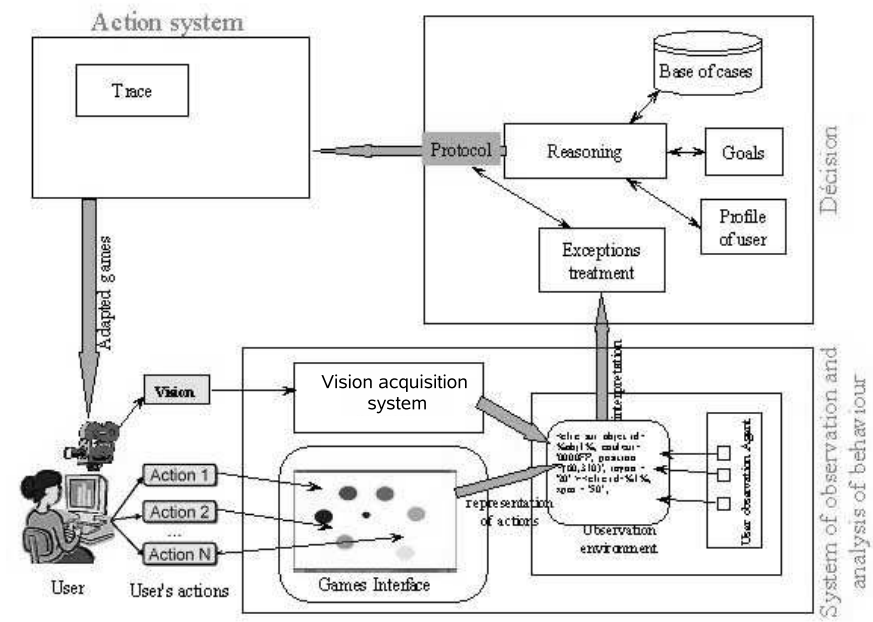

Figure 3: General architecture

- to build a protocol, that satisfies the educational goals that the child wants/must reach by taking into account his/her profile. The child's profile is defined by identity (name, date of birth, etc.), competences ([levelof-acquisition, high], [perception, medium], etc.) and preferences ([session duration, $5 \mathrm{mn}]$, [colour, green]...) $([4])$;

- to observe the child's actions during the session in order to understand his behaviour. The observation is made on the actions carried out on the peripherals: mouse, touch screen, keyboard... and by cameras for the gestures, movements, eyes orientations, attention (we will explain this part in detail in the next section) ;

- by observing the child's behaviour, the system detects cases where the activities suggested do not answer to standard gait and consequently it updates the protocol.

Figure 3 gives the general architecture of the system. We have listed three processes :

- system of observation and analysis of behaviour,

- decision system,

- action system.

The first ensures the process of observation which consists of observing, analyzing and interpreting the behaviour of the child. The second consists of adapting and checking the execution of games. Finally, the third consists of running games. For the two last systems, readers may refer to [3] or [4]. The first system is presented in the next subsection.

\subsection{Vision system and attention analysis}


Improve the understanding of the child's behavior may help to avoid rupture and avoidance, but also to maintain his attention. The understanding process can be divided into two main parts :

- acquisition and learning : during this step, each child is filmed and an expert defines characteristic behaviors from these sequences. These labels enable to understand possible links between (re)action and stimulus.

- supervision and interpretation : on the second step once again, each child is filmed. In this case, his behavior and his attention are analyzed.

For our application, we use a tracking system that does not require the subject to wear a tracking device. There are no helmets or wires to distract, influence or irritate the young subject. Instead a video camera, built into a small desk observes the child (cf. Figure 4). For the calibration, reference points are established by having the child watch animations (cf. Figures 5 and 6 ). While the tracking systems observes the child's face, eyes are located in the video image and computers record further eye movement, but also face position.

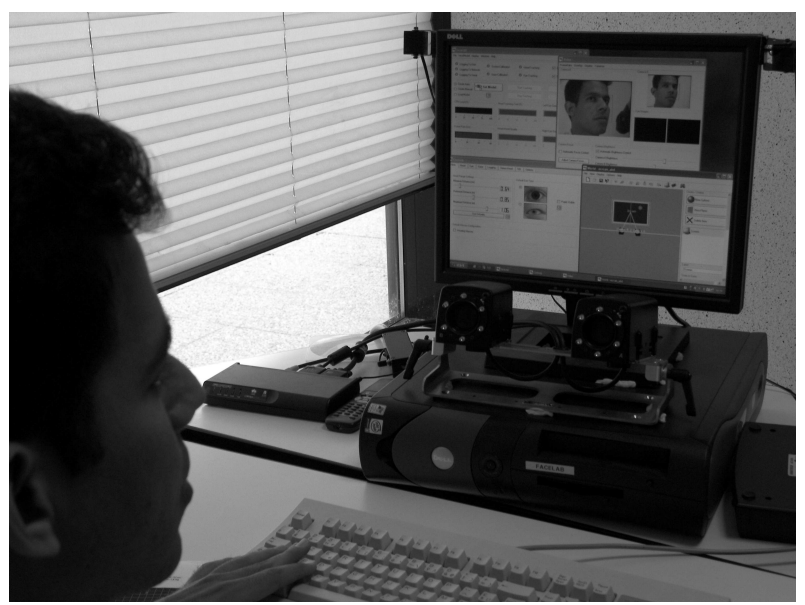

Figure 4: System used to analyse gaze and face orientation.

This allows experts to see where and for exactly how long the child is looking at the screen. The child faces, objects, and actions, either live or on video thus permit measurement of an index of attention and behavior. The fact that children do not have any physical contact with the eye tracking system and do not have to wear any special apparatus makes it a great tool especially for very young children, whether they have autism or not.

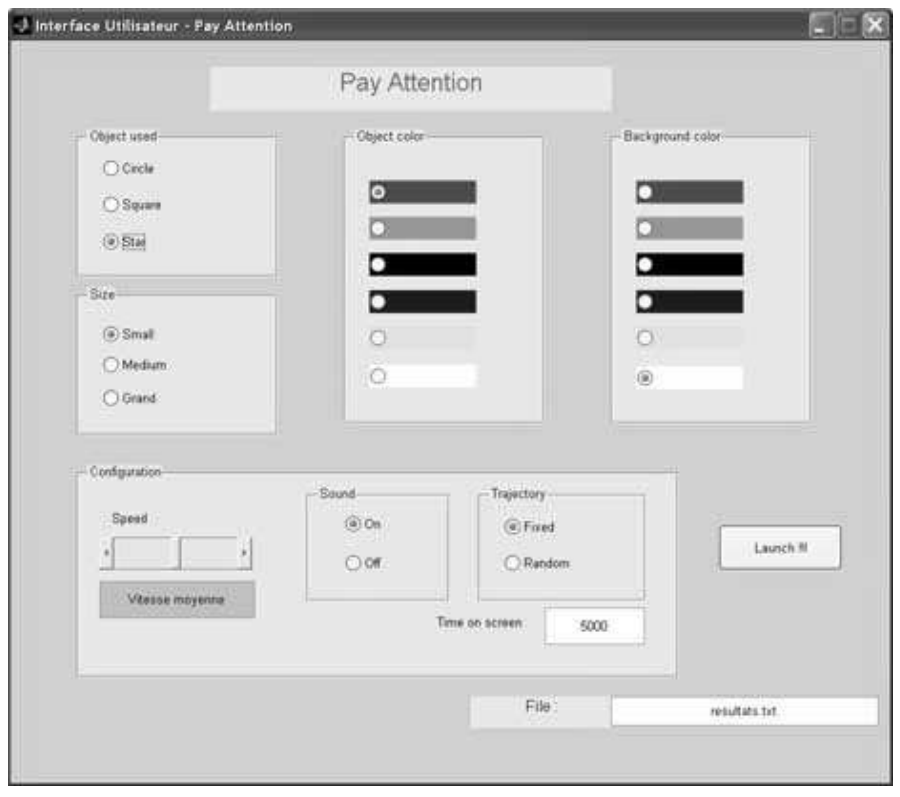

Figure 5: Interface to determine the stimuli used to focus attention and calibrate system

\section{EXPERIENCE AND RESULTS \\ 4.1 Methodology}

The main objective of this part is to determine a first measure of attention. After labelling different game sessions, we proposed a robust measure based on the adequation between gaze and object. For example, The expert asks the child to look at and follow an image of object defined a priori (a star for example). He displays this object moving along a specified trajectory (an ellipse for example), and the system automatically looks at the gaze direction of the child (cf. Figure 7). If gaze and object trajectories are correlated we can consider that the child is attentive. Thanks to expert recommendation, the systems then plays for instance a sound to focus the child's attention once again. During a real game session, we make the correlation between an object manipulated by the child and the child's gaze. In the next section, we explain in detail how we compute an attention measure.

\subsection{A robust measure of attention}

Attention analysis problem is closely correlated to the ability to maintain the interest of the child. For this reason, a suggested attention measure must be robust and adapted to each child. After labelling a series of movies presenting playing child, experts are able to roughly quantify the time $t$ after how many seconds child is inattentive, when they are not looking at the screen. Once this parameter is known, we integrate during this time the distance between gaze and region of interest. When this distance is lower than a certain percentage $p_{1}$ (adapted to each child) we estimate that the child is less attentive, when it drops under another percentage $p_{2}$, we consider that the system has to refocus the child's attention. If the distance integration measure remains for a long time between $p_{1}$ and $p_{2}$, then we also estimate that the child os less attentive. As a consequence, the system has to 


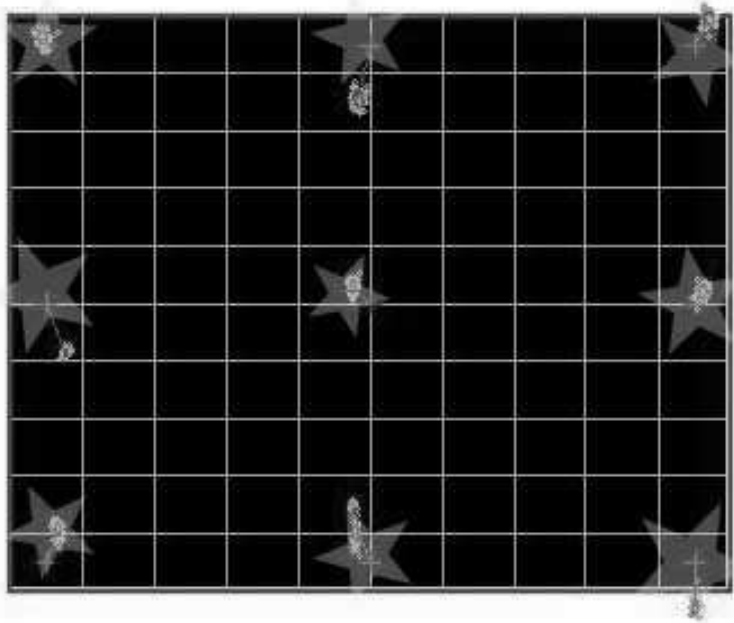

Figure 6: Superposition (in time and space) of stimulus (stars) used to calibrate and gaze detection measures (small dots behind stars)

focus child's attention. The values of algorithm parameters are defined by the expert. He analyses attention curves (cf. Figure 8 according to the bahavior of the child an experience session. Thus, these values are adapted to each child and can varied during time. The autism experts wants a manual adaptation to take into account the complexity of behaviors and the evolution of game objectives. All data are logged for the expert. Figure 8 presents the variation of our attention measure for a child after a game session. Traffic light is presented to the expert as real time tendency.

\subsection{Child's profile}

The child's profile has multiple functionalities, used at various moments, particularly in the definition of parameters previously mentioned. It also involves the interpretation of the childs actions, and the appropriate stimuli. Several types of information concerning the child are present:

- General information

- Domain knowledge

- Preferences

- History

The general information includes the child identity such as name, identifier of the child group ; the child's preferences and domain knowledge give a description of the child's profile. The history is a diary of activities suggested by the system and the results gotten by child. The history allows

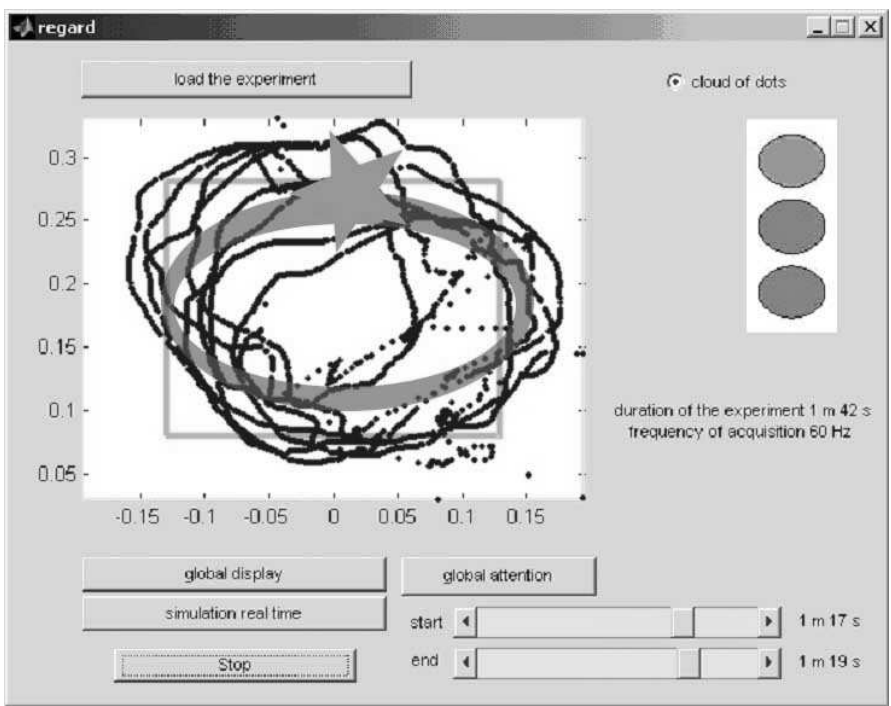

Figure 7: Superposition (in time and space) of moving stimulus (stars on an ellipse) and gaze detection measures (dots behind stars). This screen is the one presented to the expert at the end of a game session.

tracking of the evolution of the child. In order to give an interest of the history, the expert can visualize some experience or results called trace (cf. Figure 8 or 9). It concerns the child-activities interaction. The trace allows the expert to draw conclusions and adapt the defined protocols. He can also modify the directives, resources or functioning rules.

\subsection{Preliminary results}

The obtained results are interesting and promising. They are already improved in different ways. The first one is the evolution of parameters following time : actually, attention decreases as time passing by. Another example is the definition of a child's profile, in which we can store many parameters as $t$ and $p$. Our system will be tested in the Child Psychiatry of La Rochelle hospital. Meanwhile, we have applied it with children in order to define and analyze the attention measure according to various profiles. Our system is reliable to define attentive and inattentive feature of a child. Further more works and tests should and could be realized on autistic children to conclude on the effectiveness of our system.

\section{CONCLUSION AND FUTURE WORKS}

This article addresses attention analysis issues that are relevant in the AutiSTIC project which aims at improving computer games, for diagnosis and training of the children with autism and accompanying mental disorders. This approach is put in the broader context of interactive environments whom computer games are a special case. The characteristic of our approach is that the system has the capability of user adaptation. The user adaptation is based on models of user interactions, knowledge of therapists and case-based reasoning paradigm.

We have presented a system which reacts in a dynamic way thanks to the observation and analysis of behavior. The 


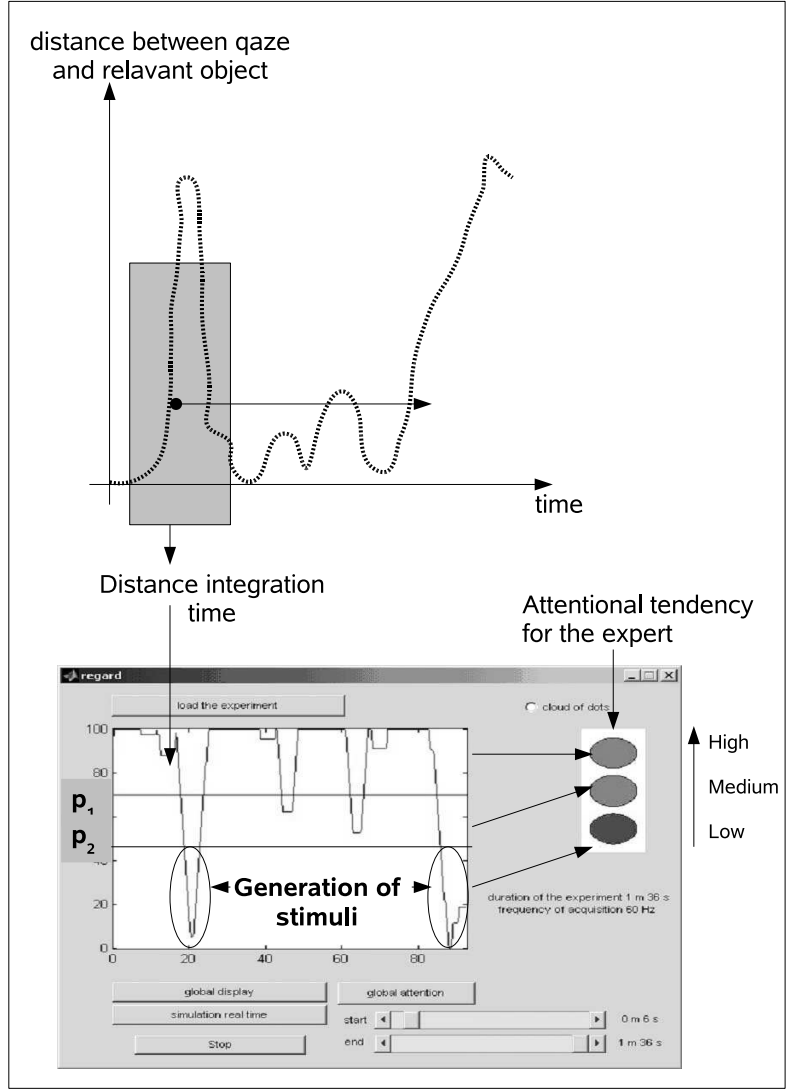

Figure 8: Evolution during a game session of the children attention using robust measure. Lines represents for the children concerned two thresholds define by the expert.

principle of architecture lies on the optimization of the extraction of information according to the context. The observation and the analysis of behavior consist in determining the behavior of child from their actions, by taking into account experts directive and the child's profile. The model that proposes is an adaptive approach that takes into account the experts directives in an educational context. The modelling of this approach uses model of the knowledge of experts, the user profile and the dynamic of their interactions.

The obtained results are interesting and promising. However, more experiments are needed to validate the proposed models and the system. The current prospects of such works are mainly centered on the improvement of the attention measure. The system we have developed is currently tested in the child psychiatry of La Rochelle hospital.

\section{ACKNOWLEDGMENTS}

The authors would like to thank D. Lambert Head of Department of Child Psychiatry of La Rochelle hospital (France) and his team, in particular : E. Bouchaud and V. Gabet for their useful suggestions regarding the rehabilitation methods of the autistic children.

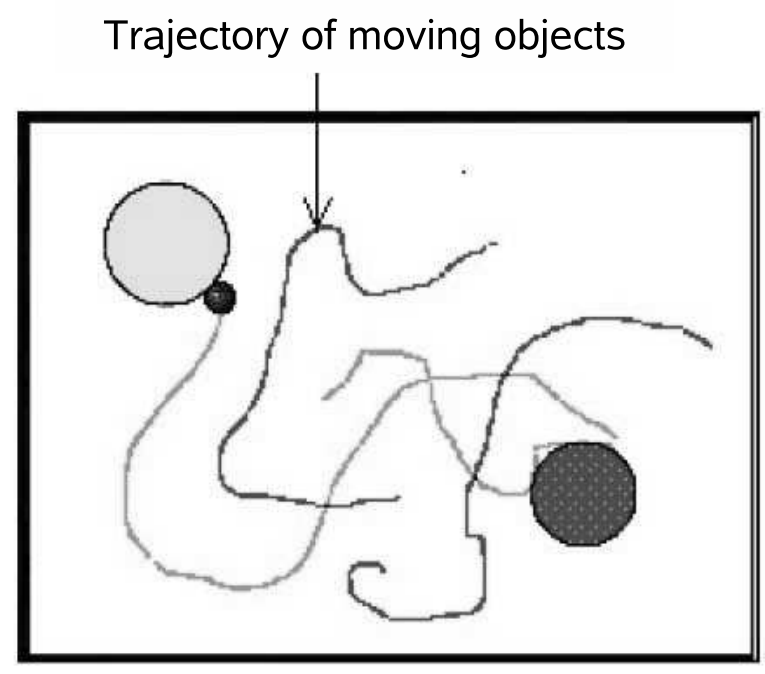

Figure 9: Exemple of execution trace of a game session.

\section{ADDITIONAL AUTHORS}

Additional authors: Dc Lambert, (email: annie.ruas@ch-larochelle.fr) Department of Child Psychiatry - Hospital of La Rochelle, U.P.E.A. Centre Hospitalier Marius Lacroix, 208, rue Marius Lacroix, 17000 La Rochelle, France.

\section{REFERENCES}

[1] Fred R. Volkmar. Handbook of Autism and Pervasive Developmental Disorders. 3th edition, 2005.

[2] Hossein Mobahi and Karrie G. Karahalios. Hci applications for aiding children with mental disorders. ACM Crossroads, Special Issue on Human-Computer Interaction, 12(2):8-12, Winter 2005.

[3] Karim Sehaba Vincent Courboulay and Pascal Estraillier. Interactive system by observation and analysis of behaviour for children with autism. Technology and Disability, 2006.

[4] K. Sehaba, P. Estraillier, and D. Lambert. Interactive educational games for autistic children with agent-based system. 4th International Conference on Entertainment Computing, page 422 432, September 2005.

[5] R.J. Reichler E. Schopler and M. Lansing. Stratgies ducatives de lautisme et des autres troubles du dveloppement. Collection Mdecine et psychotherapie, 1988.

[6] Victoria Shea Gary B. Mesibov and Eric Schopler. The TEACCH Approach To Autism Spectrum Disorders. December 2004.

[7] Mostert M.P. Facilitated communication since 1995: a review of published studies. Journal of Autism and Developmental Disorders, 31(3):287-313, 2001. 
[8] C.Grynfeltt S. Gepner, B. Deruelle. Motion and emotion: A novel approach to the study of face processing by young autistic children. Journal of Autism and Developmental Disorders, 31(1):37-45, February 2001.

[9] M. Fabri and D.J. Moore. The use of emotionally expressive avatars in collaborative virtual environments. In Proceeding of Symposium on Empathic Interaction with Synthetic Characters, held at Artificial Intelligence and Social Behaviour Convention 2005 (AISB 2005), University of Hertfordshire, 2005.

[10] K. Dautenhahn. Design issues on interactive environments for children with autism. In Proceeding 3rd Intl Conf. Disability, Virtual Reality \& Assoc, page 153159, Alghero, Italy, 2000.

[11] J. Montemayor. Physical programming : tools for kindergarten children to author physical interactive environments. Thse de doctorat, department of computer science, University of Maryland, USA, 2003.

[12] R. te Boekhorst B. Robins, K. Dautenhahn and A. Billard. Effects of repeated exposure of a humanoid robot on children with autism. In Universal Access and Assistive Technology (CWUAAT), Cambridge, UK, Mars 2004.

[13] S. Parsons. Social conventions in virtual environments: Investigating understanding of personal space amongst people with autistic spectrum disorders. Robotic $\mathbb{E}$ Virtual Interactive Systems in Autism Therapy, 2001.

[14] N. Oudin O. Grynszpan, J.-C. Martin. Towards a methodology for the design of humancomputer interfaces for persons with autism. ACM Crossroads, Special Issue on Human-Computer Interaction, Winter 2005.

[15] Thomas E. Brown. Attention Deficit Disorder: The Unfocused Mind in Children and Adults. Yale University Press, September 2005. 